Revue d'histoire de l'Amérique française

REVUE D'HISTOIRE DE L'AMÉRIQUE FRANÇAISE

\title{
L’émergence de politiques nutritionnelles au Québec, 1936-1977
}

\section{François Guérard}

Volume 67, numéro 2, automne 2013

URI : https://id.erudit.org/iderudit/1027646ar

DOI : https://doi.org/10.7202/1027646ar

Aller au sommaire du numéro

Éditeur(s)

Institut d'histoire de l'Amérique française

ISSN

0035-2357 (imprimé)

1492-1383 (numérique)

Découvrir la revue

Citer cet article

Guérard, F. (2013). L'émergence de politiques nutritionnelles au Québec, 1936-1977. Revue d'histoire de l'Amérique française, 67(2), 165-192.

https://doi.org/10.7202/1027646ar

\section{Résumé de l'article}

Un virage dans les politiques sanitaires au Québec s'amorce avec l'apparition en 1936 d'une Division de la nutrition au ministère de la Santé. Une quarantaine d'années plus tard, le gouvernement énonce une première politique de nutrition. Entre temps, il est généralement considéré que les organismes d'hygiène publique ont tardé à s'ajuster à la révolution épidémiologique faisant passer les maladies chroniques à l'avant-scène. Nous entendons revenir sur cette assertion en examinant les mesures gouvernementales adoptées dans le domaine de la nutrition. Quels moyens, de quelle envergure, sont utilisés pour convaincre les Québécois de modifier leurs habitudes alimentaires, et sur quelles conceptions reposent-ils? 


\title{
L'émergence de politiques nutritionnelles au Québec, г936-г977
}

\author{
François GuÉrard \\ Département des sciences humaines \\ Université du Québec à Chicoutimi
}

\begin{abstract}
RÉSUMÉ • Un virage dans les politiques sanitaires au Québec s'amorce avec l'apparition en 1936 d'une Division de la nutrition au ministère de la Santé. Une quarantaine d'années plus tard, le gouvernement énonce une première politique de nutrition. Entre temps, il est généralement considéré que les organismes d'hygiène publique ont tardé à s'ajuster à la révolution épidémiologique faisant passer les maladies chroniques à l'avant-scène. Nous entendons revenir sur cette assertion en examinant les mesures gouvernementales adoptées dans le domaine de la nutrition. Quels moyens, de quelle envergure, sont utilisés pour convaincre les Québécois de modifier leurs habitudes alimentaires, et sur quelles conceptions reposent-ils?
\end{abstract}

ABstract - In 1936, a shift in Quebec health policies occurred with the creation of a Nutrition Division in the Department of Health. Some forty years later, the government enunciated its first Nutrition Policy. It is generally felt that between these two events, public health organizations were slow to cope with the epidemiologic revolution that was characterized by an increase of chronic diseases. This article aims at revisiting this perception by examining the governmental measures then adopted in the field of nutrition. What were the means used to convince Quebecers to modify their eating habits? On what scale was this done? What was the conceptual framework on which this work was based?

1. Cet article a bénéficié d'un financement du Conseil de recherches en sciences humaines du Canada ainsi que de l'aide d'Aline Charles concernant une question relative aux professions de la nutrition. 
$\mathbf{L}^{\mathrm{e}}$ e Québec se dote d'une politique de la nutrition en $1977^{2}$. Quarante ans plus tôt, un premier service provincial dédié à la nutrition avait entamé ses activités au sein du ministère responsable de la santé. Entre ces deux moments, surtout partant de l'après-guerre, il est souvent considéré que les organismes d'hygiène publique ont tardé à ajuster leurs programmes à la révolution épidémiologique en cours, caractérisée par la montée des maladies chroniques dans le paysage sanitaire et par le recul des maladies infectieuses. Nous entendons revenir sur cette assertion en examinant la promotion de saines habitudes de vie, plus précisément concernant l'alimentation, effectuée au Québec de la Crise jusqu'aux années 1970 à l'instigation d'agences gouvernementales.

Quelles actions sont entreprises par les gouvernements, quelles ressources et méthodes sont mises en œuvre afin d'amener la population à se nourrir autrement? Les programmes d'action se limitent-ils à un effort éducatif ou s'y ajoute-t-il d'autres types de démarches, à caractère socioéconomique par exemple? Quels segments de la population cherche-t-on à convaincre, et suivant quelle argumentation? Cette dernière est-elle imprégnée de considérations nationalistes ou religieuses comme l'enseignement en diverses matières? Aurait-elle au contraire délaissé les appels aux valeurs collectives pour promouvoir plutôt le bien-être de l'individu? Les intentions énoncées à l'époque et la nature des interventions gouvernementales relèvent-elles de préoccupations assez proches de celles d'aujourd'hui? Autant de questions qu'il a été possible d'examiner à l'aide essentiellement mais non exclusivement d'une documentation gouvernementale. Les rapports annuels publiés par les ministères concernés livrent un résumé utile des actions entreprises, quoique partial puisqu'il met l'accent sur le travail accompli bien plus que sur ses lacunes. Les archives de la Division de la nutrition du Québec permettent d'aller au-delà de l’image publique ainsi véhiculée en ouvrant une fenêtre sur l'organisation interne de la Division et sur ses communications avec les individus, groupes et organisations intéressés par l'alimentation de la population. Les brochures publiées par les agences gouvernementales ainsi que divers articles de revues et de journaux donnent prise sur les recommandations adressées à celle-ci.

L'élaboration des politiques nutritionnelles par les gouvernements est liée à des mouvements d'idées et à des pressions issues de divers groupes

2. (Québec, province) Service des études épidémiologiques, Une politique québécoise en matière de nutrition, Québec, Ministère des Affaires sociales, direction des communications, 1977, 89 p. 
et organisations dont l'étude requiert la consultation d'autres corpus. Les nutritionnistes et médecins, certains organismes caritatifs, des éducateurs religieux et laïques, les naturopathes et autres praticiens de médecines parallèles à partir des années 1960, les producteurs agricoles, l'industrie alimentaire et des associations de consommateurs apparues dans la décennie 1970, toute une gamme d'acteurs entreprennent des démarches pour obtenir des réorientations aux politiques nutritionnelles durant la période couverte. Une étude a ainsi fait ressortir le lobbying d'un organisme pionnier dans le domaine de l'aide et de l'éducation alimentaires, le Montreal Diet Dispensary, auprès du gouvernement fédéral dans les décennies 1930 et $1940^{3}$. Et nos sources montrent que le même organisme interviendra, auprès du gouvernement provincial cette fois, lorsque se discuteront les réformes de la Révolution tranquille ${ }^{4}$. Les politiques nutritionnelles sont adoptées en aval de toutes ces pressions, dont il serait toutefois prématuré d'évaluer les poids respectifs dans les prises de décisions, avant que suffisamment d'études aient mis en lumière l'action des groupes ou organisations concernés. Aussi cet article abordera-t-il l'émergence de politiques nutritionnelles à travers les programmes mis en œuvre, sans prétendre épuiser les facettes de leur genèse.

Seront successivement abordés les reproches adressés, à partir de la Révolution tranquille, aux politiques d'hygiène publique de l'après-guerre, puis les principaux organismes publics actifs dans le domaine de la nutrition, les actions entreprises dont le déploiement d'une panoplie de mesures éducatives, les types d'arguments choisis pour convaincre et la conception d'une nouvelle nutrition publique dans les années 1970.

\section{DES POLITIQUES PRÉVENTIVES CRITIQUÉES PUIS OUBLIÉES}

Au moment de la Révolution tranquille, les politiques d'hygiène publique sont soumises à des critiques sévères, alors que les efforts consacrés à la prévention sont jugés nettement insuffisants et mal organisés. Dans le prolongement de cette perception, et vraisemblablement convaincus que l'hygiène publique de l'après-guerre n'offre que peu de nouveauté et par

3. Yolande Cohen, «De la nutrition des pauvres malades: L'histoire du Montreal Diet Dispensary de 1910 à 1940 ", Histoire sociale/Social History, 41, 81 (mai 2008): 133-163.

4. Lettre d'Agnes C. Higgins, directrice du Montreal Diet Dispensary à l'honorable Victor C. Goldbloom, 3 juillet 1970, Bibliothèque et Archives nationales du Québec (BAnQ), Ministère de la Santé et des Services sociaux (MSSS), Bureau du sous-ministre, Montreal Diet Dispensary, 7B012 02-04-001B01/1970; lettre de Roger Marier, sous-ministre de la Famille et du Bien-être social à Agnes C. Higgins, 22 septembre 1970, même localisation. 
conséquent d'intérêt, les chercheurs historiens ont tardé à l'aborder, hors quelques exceptions ${ }^{5}$.

Une excellente illustration du désaveu pesant sur les mesures préventives au début des années 1970 réside dans la suppression des unités sanitaires. Chargées de l'essentiel des interventions directes auprès de la population hors de Montréal, leurs responsabilités seront transférées à de nouveaux organismes parce qu'elles sont alors jugées incapables de s'adapter aux transformations récentes ou souhaitées du monde sanitaire, et notamment de refondre leur action pour combattre efficacement les maladies chroniques ${ }^{6}$. Ainsi, d'après la Commission d'enquête sur la santé et le bien-être social (CESBES) en 1970, la prévention pratiquée par les unités a été "reléguée $[. .$.$] au rang de sous-service», et "n’atteint pas suf-$ fisamment la masse de la population »; le travail y est dévalorisé, "routinier et peu stimulant», ce qui engendre des difficultés de recrutement de personnel liées au "manque d'intérêt des professionnels de la santé, des établissements de santé et même des gouvernements " ${ }^{7}$. Même son de cloche en 1971 lorsque le Comité d'étude sur la prévention sanitaire, nommé par le gouvernement du Québec, écrit: «le système des unités sanitaires se détériorant depuis une quinzaine d'années, est maintenant dans un état lamentable ${ }^{8}$ ".

Quant au secteur de la nutrition, la condamnation paraît au moins aussi sévère, quoique tacite : la CESBES, là où elle traite d'alimentation, ne fait aucune mention du service du ministère de la Santé dédié à la nutrition et tout juste démantelé ou en voie de l'être ${ }^{9}$, affirme qu'il n'existe pas de recherche sur l'alimentation hors de Montréal alors que ledit service avait multiplié de telles enquêtes des années 1930 jusqu'aux années $1960^{10}$, et

5. Quelques travaux font exception: Georges Desrosiers, Benoît Gaumer et Othmar Keel, La santé publique au Québec: Histoire des unités sanitaires de comté: 1926-1975 (Montréal, Presses de l'Université de Montréal, 1998); B. Gaumer, G. Desrosiers, O. Keel, Histoire du service de santé de la ville de Montréal $1865-$ 1975 (Québec, Les éditions de l'IQRC, 2002).

6. Georges Desrosiers et Benoît Gaumer, Jean Grégoire. Médecin et administrateur-Premier sous-ministre de la Santé du Québec (Montréal, LIDEC, 2012), 54.

7. (Québec, province) Commission d'enquête sur la santé et le bien-être social (Castonguay-Nepveu), Rapport de la Commission d'enquête sur la santé et le bien-être social, deuxième partie, La santé, Tome I La situation actuelle (Gouvernement du Québec, 1970), 103-104.

8. (Québec, province) Comité d'étude sur la prévention sanitaire (Corbett MacDonald), Rapport du comité d'étude sur la prévention sanitaire (Gouvernement du Québec, 1971), 4.

9. Un rapport du Service de la nutrition est publié dans le dernier rapport annuel du ministère de la Santé pour l'année 1969. La disparition de ce dernier, avec la création du ministère des Affaires sociales en 1970, s'accompagne d'une réorganisation des services.

10. Cet aspect de l'activité de la Division de la nutrition est documenté dans les archives conservées à BAnQ, de même que par divers articles publiés. Voir notamment: BAnQ, MSSS, Enquêtes sur la nutrition, 7B012 01-02-005A-01, J.-Ernest Sylvestre, La consommation du lait dans certains comtés de la province 
préfère se référer à des communications présentées par des tiers lors de congrès récents, voire à un compte rendu de livre paru dans un quotidien, plutôt qu'aux rapports et publications du service ${ }^{11}$.

Il en est ressorti une image sclérosée de l'hygiène publique d'aprèsguerre, caractérisée par un immobilisme qui tranche sur les décennies antérieures faites de gains et de conquêtes, alors qu'à l'échelle internationale, l'hygiène publique serait mal parvenue à redéfinir son action dans un contexte épidémiologique changeant et aurait perdu en autonomie ${ }^{12}$. Cela explique en partie que les chercheurs se soient grandement intéressés à la période en quelque sorte glorieuse de mise en place des appareils d'hygiène publique jusqu'à la Seconde Guerre mondiale, mais beaucoup moins à ce qui a suivi et qui paraissait plutôt terne. Entre, d'une part, les programmes de lutte contre la mortalité infantile et la tuberculose de la première moitié du siècle, et d'autre part les campagnes antitabagiques des décennies 1970 et 1980, l'hygiène publique paraissait avoir eu peu à dire ou à faire valoir, être demeurée stationnaire. Cela était amplifié au Québec par le discrédit rattaché à l'État québécois d'avant la Révolution tranquille, les réformateurs ayant légitimé leur action en partie par la dénonciation d'un retard de l'évolution des institutions du Québec. Dans cette optique, la présomption que le gouvernement aurait tardé à mettre en place les mesures requises pour contrer la progression des maladies chroniques ne constituait qu'une marque d'inertie supplémentaire, voire de passéisme, parmi tant d'autres.

Il semble bien qu'à l'époque, les organismes d'hygiène publique n'aient effectivement pas mis rapidement en place des mesures ou des programmes vraiment efficaces pour en arriver à infléchir les comportements durables des populations liés à la santé et plus particulièrement aux maladies chroniques. Mais il faut bien admettre aussi qu'ils y peinent encore aujourd'hui, même si certains succès ont été enregistrés notamment pour

d'après les renseignements fournis par une enquête faite en 1937, Rapport, Ministère de la Santé, Division de la Nutrition, $1^{\text {er }}$ mai 1939; J.-Ernest Sylvestre et Honoré Nadeau, «Enquête sur l'alimentation habituelle des familles de petits-salariés dans la ville de Québec», The Canadian Public Health Journal (mai 1941): 241-250 ; J.-E. Sylvestre et L. B. Pett, «Enquête alimentaire chez les écoliers des comtés de Lévis et de Brôme-Missisquoi, Québec», Bulletin sanitaire, 49, 3 (juillet-septembre 1949): 45-59; J.-Ernest Sylvestre, «Nos écoliers sont-ils bien nourris?», L’Union médicale du Canada (avril 1947): 399-412; BAnQ, MSSS, Enquêtes sur la nutrition, 7 B 012 06-05-002A-01/1951-54, rapport Enquête alimentaire. Canadian Carborundum Company Limited, Shawinigan Falls, Co. St-Maurice; BAnQ, MSSS, Enquêtes sur la nutrition, 7 B 012 06-05-002A-01 / 1960-63, J.-Ernest Sylvestre, rapport Le problème du lunch, 1963.

11. (Québec, province) Commission d'enquête (Castonguay-Nepveu), Rapport ... 1970, op. cit., 57-59.

12. Voir Dorothy Porter, Health, Civilization, and the State: A History of Public Health From Ancient to Modern Times (New York, Routledge, 1999), 290. 
la réduction du tabagisme. Qu'il suffise, pour bien camper la difficulté de la tâche, de rappeler qu'en ce qui a trait aux habitudes alimentaires génératrices de surplus de poids, actuellement sources des pires inquiétudes, des cris d'alarme ont été lancés au Canada et au Québec dès les années 1970 et même avant ${ }^{13}$, donc depuis au moins une quarantaine d'années, sans que l'on soit parvenu depuis à contenir cette hausse pondérale. Autre exemple, depuis les années 1940, les responsables sanitaires gouvernementaux répètent sans discontinuer que les Québécois ne mangent pas assez de fruits, de légumes et de produits céréaliers. Quoique relayé à la population pendant environ 70 ans, ce message n'a rien perdu de son actualité puisqu'il demeure encore aujourd'hui au menu des nutritionnistes. De façon plus large, si les organismes d'hygiène publique d'aprèsguerre n'ont su faire des Québécois des consommateurs respectueux du guide alimentaire canadien, comme le répètent les divers rapports de recherche de la Division de la nutrition, cela vaut pour leurs successeurs et pour les mesures mises en place depuis.

Du reste, les auteurs actuels, traitant des méthodes à adopter pour amener les populations à transformer leurs habitudes de vie reliées à la santé, reconnaissent la difficulté de la tâche, et continuent d'en débattre. Si certains considèrent que les habitudes de vie relèvent essentiellement de la capacité de l'individu à faire ses choix de consommation de façon autonome vis-à-vis de contraintes diverses, d'autres mettent au contraire l'accent sur des déterminants structurels, externes à l'individu. Les premiers misent sur l'éducation pour remodeler les habitudes de vie, les seconds estiment qu'il faut y ajouter des transformations de l'environnement social, culturel et économique dans lequel l'individu prend ses décisions ${ }^{14}$. Dans cette dernière

13. La compagnie Metropolitan Life Insurance (MLI) publie dès les années 1950 des brochures signalant les risques associés à l'obésité et les rapports annuels du ministère de la Santé nationale et du Bien-être social (MSNBES) au gouvernement fédéral mentionnent les problèmes d'obésité dans les années 1960. Une enquête du MSNBES sonne vraiment l'alarme dans la décennie suivante. Voir MLI, Direction générale au Canada, Obésité et maigreur (Ottawa, MLI, juin 1953), 32 p. ; MLI, Quel est votre poids normal? (Ottawa, MLI, Direction générale au Canada, 1957), 4 p.; (Canada) MSNBES, Rapport annuel pourl'année financière terminée le 31 mars 1964 (Ottawa, Imprimeur de la Reine et Contrôleur de la Papeterie, 1965), p. 77; (Canada) Bureau des sciences de la nutrition, Nutrition Canada: compte-rendu de l'étude menée au Québec (Ottawa, MSNBES, 1975), 172 p.

14. Concernant ces débats sur les habitudes de vie, voir William C. Cockerham, "Health Lifestyle Theory and the Convergence of Agency and Structure ", Journal of Health and Social Behavior, 46, 1 (mars 2005): 51-67; Deborah A. Cohen, Richard A. Scribner et Thomas A. Farley. «A Structural Model of Health Behavior: A Pragmatic Approach to Explain and Influence Health Behaviors at the Population Level", Preventive Medicine, 30 (2000): 146-154; Amy Schulz et Mary E. Northridge, "Social Determinants of Health: Implications for Environmental Health Promotion», Health Education \& Behavior, 31 (2004): 455-471; Organisation de coopération et de développement économiques (OCDE), L'obésité et l'économie de la prévention: Objectif santé (Paris, Éditions OCDE, novembre 2010), 285 p. 
perspective, l'Organisation mondiale de la santé en 2004 et le Conseil de la Science et de la Technologie du Québec en 2007 ont émis l'avis que les habitudes de vie sont grandement influencées par le milieu, et que les politiques antérieures n'en ont pas assez tenu compte ${ }^{15}$. L'idée n'est pourtant pas nouvelle qu'il faille jouer à la fois sur l'éducation et l'environnement, mais les politiques sanitaires auraient peu touché au second ${ }^{16}$. Il est à noter toutefois que les programmes de revenu minimum mis en place durant la Révolution tranquille et d'autres mesures sociales caractéristiques de l'Étatprovidence constituent déjà une intervention majeure réduisant l'emprise des contraintes externes à l'individu.

Au sortir de la Seconde Guerre mondiale, les responsables sanitaires prenaient acte de la transition épidémiologique des maladies infectieuses vers les maladies chroniques. Les moyens idoines d'intervention restaient à définir, et si des appels à des mesures sociales relevant la capacité d'acheter les aliments requis se faisaient alors entendre, les voix privilégiant l'éducation de la population étaient plus puissantes ${ }^{17}$. Quelles que soient les voies d'action choisies, il fallait en outre convaincre les décideurs politiques d'affecter des ressources à une toute nouvelle lutte sanitaire. Pour les luttes précédentes, cela ne s'était pas fait si facilement, ni si rapidement, ce dont rendent compte de nombreuses études ayant mis en lumière les requêtes incessantes des partisans de l'hygiène publique au fil des décennies et les hésitations des autorités gouvernementales ou municipales ${ }^{18}$.

Bref, avec le recul, alors que les spécialistes discutent toujours des meilleures façons d'amener la population à modifier ses habitudes de vie et que les gouvernements mettent en branle de grands programmes de promotion $^{19}$, il paraît pertinent de s'interroger sur l'activité des orga-

15. Organisation mondiale de la Santé, Stratégie mondiale pour l'alimentation, l'exercice physique et la santé (Genève, Organisation mondiale de la Santé, 2004), 19; (Québec, province) Conseil de la science et de la technologie, Défi habitudes de vie: Stratégie de recherche et de transfert (Québec, Conseil de la science et de la technologie, 2007), 3, 10.

16. Michel O’Neill, Sophie Dupéré, Ann Pederson et Irving Rootman dir., Promotion de la santé au Canada et au Québec, perspectives critiques (Québec, Les Presses de l’Université Laval, 2006), 6-11 et 16-19.

17. C'est l'argument central de Ian Mosby dans «Making and Breaking Canada's Food Rules: Science, the State, and the Government of Nutrition, 1942-1949», dans Franca Iacovetta, Valerie J. Korinek et Marlene Epp, dir., Edible Histories, Cultural Politics (Toronto, University of Toronto Press, 2012), 409-432.

18. Pour le Québec, voir François Guérard, «La formation des grands appareils sanitaires, 1800-1945 », dans Normand Séguin, dir., Atlas historique du Québec. L’institution médicale (Québec, Les Presses de l’Université Laval, 1998), 75-115.

19. Brigitte Lachance, Martine Pageau et Sylvie Roy, Investir pour l'avenir : plan d'action gouvernemental de promotion des saines habitudes de vie et de prévention des problèmes reliés au poids 2006-2012 (Québec, Santé et services sociaux Québec, 2006), 50 p. 
nismes d'hygiène publique lors des décennies 1940 à 1970, consécutive à la prise de conscience de problèmes alors émergents, aujourd'hui prééminents, et de le faire en privilégiant la promotion de saines habitudes alimentaires. Nous pensons que si l'orientation dominante au Québec durant cette période au sein des organismes publics était celle de l'éducation, et ce, dans le prolongement de la New Public Health des années 1910 et 1920 qui déjà misait sur une responsabilisation des individus, leur action ne s'y limite pas. Avant la décennie 1970-1980, ils disposent toutefois de bien peu de moyens.

\section{LA DIVISION DE LA NUTRITION ET AUTRES ORGANISMES}

Divers organismes gouvernementaux fédéraux comme provinciaux ont joué un rôle dans le développement de politiques nutritionnelles, que ce soit pour la recherche, la formation de personnel qualifié ou l'éducation de la population. Comme la santé demeure une responsabilité provinciale au Canada, les organismes publics de la province de Québec ont assuré l'essentiel des contacts avec la population et les institutions locales, quoique dans certains cas avec l'appui de leurs vis-à-vis fédéraux ou dans le prolongement de programmes fédéraux. C'est de leur action qu'il sera prioritairement question, bien que le gouvernement fédéral ait exercé un leadership par la définition des normes alimentaires canadiennes ${ }^{20}$, la conception d'outils de recherche et la publication d'imprimés.

Un virage dans les politiques sanitaires au Québec s'amorce avec l'apparition en 1936 d'une Division de la Nutrition (Division) au sein du département de la Santé. Alors que l'action gouvernementale dans le domaine de l'hygiène publique avait jusqu'alors visé principalement le contrôle des maladies contagieuses et de la mortalité qui leur était associée, la nouvelle division va s'attaquer à d'autres types de pathologies et d'objectifs. En 1937, le gouvernement du Canada crée le Conseil canadien de la Nutrition, puis en 1941 une Division de l'Hygiène alimentaire au ministère de la Santé nationale et du Bien-être social. On le voit, la période de la Crise et les années suivantes ont été propices à l'apparition d'organismes publics dédiés à l'alimentation. En effet, la nécessité d'approvisionner en victuailles les victimes du chômage a soudainement attiré l'attention sur cet enjeu particulier: les gouvernements ont dû intégrer une aide alimentaire au programme des secours directs et une kyrielle d'organismes privés à caractère charitable ont également contribué à la distribution d'aliments et de repas. 
Devant l'incapacité temporaire d'une partie de la population de combler ses besoins les plus élémentaires, l’alimentation s'est dressée en problème national d'envergure, et le demeurera durant la guerre avec le rationnement. Le contexte appelait à la création d'organismes spécialisés.

D'autres organismes gouvernementaux interviennent parfois. C'est le cas, au fédéral comme au provincial, des ministères responsables de l'agriculture : les choix de consommation des Canadiens et des Québécois ont des répercussions sur l'écoulement de la production agricole, et la tentation est grande d'orienter ces choix afin de favoriser les cultivateurs du pays ou de la province, dans une perspective de développement économique. Ainsi, dans les années 1920, le ministère de l'Agriculture fédéral publie des brochures vantant la consommation de produits laitiers, bientôt de légumes ${ }^{21}$ (voir figure 1, p. 174). Le ministère de l'Agriculture provincial aussi s'engagera dans cette voie par la production de brochures ${ }^{22}$, ou dans les années 1970 par un programme ambitieux de distribution de lait dans les écoles, programme dont il sera question plus loin.

Sauf exception, lorsqu'il est question prioritairement de santé plutôt que d'économie, il revient à la Division de la nutrition du Québec d'agir sur le territoire provincial, parfois dans le prolongement de programmes mis en œuvre par le gouvernement fédéral ou avec son appui, le plus souvent de façon pleinement autonome. Ses activités débutent en 1937. Pendant de nombreuses années, elle sera composée d'un nombre très restreint d'employés, ce qui limitera sa capacité d'intervention. À ses débuts, la Division en compte trois : le directeur J.-E. Sylvestre, médecin diplômé en hygiène et en nutrition, une nutritionniste et une infirmière ${ }^{23}$. Jusqu'au début des années 1950, seules quelques personnes travaillent à la Division ${ }^{24}$. Mais J.-E. Sylvestre a d'autres ambitions. Lorsqu'en 1948 un

21. Helen G. Campbell, Consommons du lait (Ottawa, ministère fédéral de l'Agriculture, Division de l'industrie laitière et de la réfrigération, 1924), 30 p.; Helen G. Campbell, Consommons du fromage (Ottawa, ministère fédéral de l'Agriculture, Division de l'industrie laitière et de la réfrigération, 1928), 24 p.; Laura C. Pepper, Le lait, l'aliment par Excellence (Ottawa, ministère de l'Agriculture, 1939), 24 p. ; Édith L. Elliot, Légumes canadiens pour tous les jours (Ottawa, ministère de l'Agriculture, service des marchés, publication 521, Bulletin de la ménagère $\left.\mathrm{n}^{\circ} 6,1941\right), 21 \mathrm{p}$.

22. J. E. Grisé, Les conserves. Légumes Fruits Viandes variées en tout temps de l'année, (Québec, ministère de l'Agriculture, 1943), 63 p. ; Estelle LeBlanc, Mangeons plus de légumes : quelques recettes pratiques, (Québec, ministère de l'Agriculture, service de la publicité, bulletin n $\left.{ }^{\circ} 136,1937\right), 31$ p. Ce dernier document a été révisé et édité plusieurs fois sous divers titres jusqu’aux années 1970.

23. (Québec) ministère de la Santé et du Bien-être Social, Premier Rapport Annuel du Ministère de la Santé et du Bien-être Social pour les années 1935 à 1941 (Québec, ministère de la Santé et du Bien-être Social, 1944), 180.

24. (Québec), ministère de la Santé, Septième rapport du Ministère de la Santé pour l'année 1950 (Québec, ministère de la Santé, 1951), p. 71. 


\section{FIGURE I}

\section{Brochure publiée par le ministère fédéral de l'Agriculture}

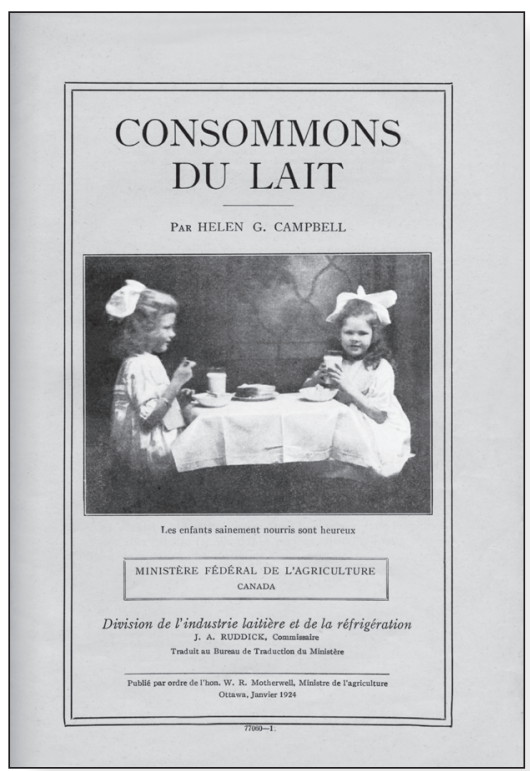

Source: Helen G. Campbell, Consommons du lait (Ottawa, ministère fédéral de l'Agriculture, Division de l'industrie laitière et de la réfrigération, 1924). comité devant enquêter sur les services de santé est mis sur pied, on le nomme président. Dans son rapport déposé en 1951, le comité indique que le service, responsable aussi de l'hygiène maternelle et infantile, comptait en 1948 deux nutritionnistes et deux sténo-dactylos en plus du directeur. Il propose une réforme du service de nutrition qui ajouterait aux $11570 \$$ de l'année 1948, un montant annuel de $68650 \${ }^{25}$. Une hausse du personnel affecté à la division survient effectivement après 1952, alors que trois nutritionnistes y travaillent ${ }^{26}$. Une diététiste est ajoutée en $1953^{27}$, puis une nutritionniste et deux diététistes en $1954^{28}$. L'année suivante, outre le directeur, huit personnes sont employées ${ }^{29}$. Au début des années 1960, le personnel
. La Division accroît donc sa est composé d'une dizaine de personnes ${ }^{30}$. La Division accroît donc sa
capacité d'action, mais c'est encore bien peu. En 1963, dans un rapport adressé au ministre de la Santé, J.-E. Sylvestre en appelle de nouveau à

25. Il est vrai que la division compte alors une section d'hygiène maternelle et infantile, mais l'essentiel des dépenses prévues concernent la nutrition. Voir (Québec, province) J.-Ernest Sylvestre, dir., Enquête sur les services de santé (1948). Tome III Services de santé provinciaux (août 1951), 221-222.

26. (Québec) ministère de la Santé, Neuvième Rapport du Ministère de la Santé pour l'année 1952 (Québec, ministère de la Santé, 1953), 73.

27. (Québec) ministère de la Santé, Dixième Rapport du Ministère de la Santé pour l'année 1953 (Québec, ministère de la Santé, 1954), 75.

28. Aujourd'hui au Québec, les titres de nutritionniste, de diététicien et de diététiste désignent la même profession. Au début des années 1950 par contre, les notions de diététiste et de nutritionniste renvoyaient à des standards de formation et de pratique distincts, quoique non réglementés jusqu'en 1956 lorsque survient la Loi des diététistes du Québec accordant un titre réservé aux membres de l’Association des diététistes du Québec.

29. (Québec) ministère de la Santé, Douzième Rapport du Ministère de la Santé pour l'année 1955 (Québec, ministère de la Santé, 1956), 82.

30. (Québec), ministère de la Santé, Dix-neuvième rapport du Ministère de la Santé, 1962 (Québec, ministère de la Santé, 1963), 81-82. 
une augmentation des ressources. Il estime que le manque de personnel explique les résultats mitigés en termes d'éducation de la population. Il ajoute que «La Division de la Nutrition n'a jamais eu assez de nutritionnistes pour entraîner les infirmières hygiénistes et les éducateurs qui, en somme, sont les personnes en mesure de faire l'éducation des adultes et des enfants surtout ${ }^{31}$.»

En somme, s'il avait fallu que la mise en ouvre de programmes éducatifs ou autres ne repose que sur les épaules du personnel de la Division, ce travail aurait été réduit à bien peu de choses pour le Québec avant la décennie 1970.

\section{LES MÉTHODES DE DIFFUSION DES RECOMMANDATIONS}

Avec ses maigres ressources, la Division se tourne dès ses débuts vers des actions à visées multiplicatrices, misant sur un transfert et une amplification par d'autres acteurs. C'est dans cet esprit que le directeur de la division écrit dans son rapport annuel de 1945: «le but principal de notre division est avant tout la direction scientifique et pratique des groupes susceptibles de quelque influence sur la population ${ }^{32} »$. Bon nombre d'activités de la Division obéissent à cette perspective par des interventions auprès de groupes ou d'organisations qui reconduiront ses avis sur les pratiques nutritionnelles les plus avantageuses, notamment par la formation de personnel apte à propager de saines recommandations. La plupart relèvent du domaine de l'éducation, mais l'une d'entre elles, permettant de conseiller des organisations pour leurs services alimentaires, vise plutôt à modifier l'environnement de l'individu.

Dans les années 1940, la Division offre déjà un service de consultation pour les entreprises et les organismes désireux d'améliorer leurs services alimentaires $^{33}$. La Division mène une courte enquête dans l'établissement puis émet des recommandations. Dans un premier temps, ce service est utilisé par des pensionnats, des collèges classiques, des colonies de vacances et quelques hôpitaux en quête de régimes convenables pour leurs pupilles ou patients. Les communautés religieuses enseignantes ou hospitalières, responsables de la plupart de ces établissements, sont alors les principales organisations recourant à l'expertise de la Division. En 1944

31. BAnQ, MSSS, Enquêtes sur la nutrition, 7 B 012 06-05-002A-01/ 1960-63, J.-Ernest Sylvestre, rapport Le problème du lunch à l'école, op. cit.

32. (Québec, province) Ministère de la Santé et du Bien-être Social, Troisième rapport du Ministère de la Santé pour les années 1944, 1945 et 1946 (Québec, Ministère de la Santé et du Bien-être Social, 1948), 296.

33. Les rapports annuels de la Division rendent compte de cette activité de consultation. 
par exemple, des leçons sont données aux religieuses de Sainte-Marthe de Saint-Hyacinthe, une communauté vouée à l'entretien des pensionnats pour garçons et qui prend en charge les repas donnés aux élèves comme au personnel ${ }^{14}$. Parfois aussi, des entreprises privées demandent de l'aide pour leurs services alimentaires destinés aux employés, comme c'est le cas en 1947 pour quatre d'entre elles ${ }^{35}$. S'il s'agit en général de concevoir des menus, certains demandent également conseil pour l'aménagement de leur cafétéria, ou veulent un avis plus général sur leurs services alimentaires. En 1953, à la faveur de l'arrivée d'une diététiste à la Division, cette dernière entreprend la mise sur pied d'un service de consultation visant spécifiquement les hôpitaux ${ }^{36}$. Toute cette activité a pour objectif que les élèves, pupilles, patients et travailleurs qui fréquentent les services alimentaires des grandes organisations y trouvent un environnement sain où faire les meilleurs choix.

Si elle n'a pas le personnel pour prendre en charge directement l'éducation de la population, la Division s'efforce de former ceux et celles en situation de le faire. Ce sont en général des professionnels de la santé. En 1951 par exemple, J.-E. Sylvestre offre un enseignement universitaire à des médecins et à des infirmières dans les murs de l'École d'Hygiène de l'Université de Montréal, et aux étudiants en médecine de l'Université Laval et de l'Université de Montréal ${ }^{37}$. À partir de cette année, une formation de dix jours en hygiène alimentaire est offerte à des infirmières spécialisées comme éducatrices hygiénistes et qui travaillent dans les unités sanitaires ${ }^{38}$. Des cours sont aussi donnés dans certaines écoles d'infirmières, à l'époque dirigées par des hôpitaux, voire à des infirmières de colonies de vacances. Les employées de la Division se déplacent également pour visiter les éducateurs religieux ou laïques dans les établissements scolaires, les pensionnats de garçons et de filles ou les établissements d'enseignement ménager. Après l'élargissement de son personnel dans la première moitié des années 1950, l'activité pédagogique de la Division prendra nettement de l'ampleur.

Toutes ces interventions auprès d'organisations éducatives qui relèvent d'autres ministères peuvent toutefois nécessiter une clarification des préro-

34. (Québec, province) Ministère de la Santé et du Bien-être Social, Troisième rapport..., op. cit., p. 284.

35. (Québec, province), ministère de la Santé, Quatrième rapport du Ministère de la Santé pour l'année 1947, (Québec, Ministère de la Santé et du Bien-être Social, 1949), 72.

36. (Québec, province) ministère de la Santé, Dixième Rapport du Ministère de la Santé pour l'année 1953 (Québec, ministère de la Santé, 1954), 75.

37. (Québec, province) ministère de la Santé, Huitième Rapport du Ministère de la Santé pour l'année 1951 (Québec, ministère de la Santé, 1952), 73-74.

38. (Québec, province), ministère de la Santé, Neuvième Rapport du Ministère de la Santé pour l'année 1952 (Québec, ministère de la Santé, 1953), 74. 
gatives de chacun. En 1942, Sylvestre explique ainsi qu’il entend garder «la main haute $[\ldots]$ sur la direction scientifique de l'enseignement ${ }^{39}$ ", et que la Division conseille à ce sujet le ministère de l'Instruction publique ainsi que la Division de l'Économie domestique au ministère de l'Agriculture.

Les enseignements donnés au personnel des unités sanitaires, une autre division du ministère responsable de la santé, sont particulièrement soignés. En effet, ce personnel sera systématiquement utilisé pour la mise en œuvre des programmes conçus à la Division. Ainsi, pour la recherche sur l'alimentation des Québécois, ce sont bien souvent les infirmières des unités, chapeautées par la Division, qui recueillent l'information dans la population, notamment dans les écoles grâce à l'aide des enseignants ${ }^{40}$. Le personnel des unités est également invité à intégrer des considérations sur l'alimentation dans tous les événements prévus pour éduquer la population à l'hygiène: conférences, séances d'information, distribution de prospectus, etc. Pour qu'il soit apte à le faire, les nutritionnistes de la Division visitent les unités les unes après les autres et y informent les médecins comme les infirmières.

Au-delà des actions visant un effet d'entraînement, d'autres moyens sont mis à profit pour rejoindre des groupes ciblés ou la population en général, que ce soit oralement ou par la distribution d’imprimés. Au fil des années, de nombreuses brochures sont publiées par diverses agences gouvernementales. L'activité éditoriale du gouvernement fédéral est nettement plus intense que celle du gouvernement provincial. Avec son personnel restreint, la Division ne peut préparer en grand nombre des feuillets éducatifs, des brochures ou des livres ${ }^{41}$.

Trois catégories de publications peuvent être distinguées selon leurs publics cibles. Certaines visent des groupes professionnels actifs en éducation ou en santé, en particulier les médecins et les infirmières mais aussi les enseignants. Dans cette catégorie, on retrouve les titres maintes fois réédités Manuel de diétothérapie pour les étudiantes infirmières ${ }^{42}$ et Considérations sur les besoins du corps humain et les règles alimentaires, destiné

39. (Québec, province) ministère de la Santé et du Bien-être Social, Deuxième rapport du Ministère de la Santé pour les années 1941, 1942, et 1943 (Québec, ministère de la Santé et du Bien-être Social, 1945), 272.

40. Dès la première enquête effectuée par la Division, les unités sanitaires sont mises à contribution. Ce sera constamment le cas par la suite comme en témoignent les rapports annuels du ministère de la Santé du Québec. Voir BAnQ, MSSS, Enquêtes sur la nutrition, 7B012 01-02-005A-01, J.-Ernest Sylvestre, La consommation..., op. cit., 1 .

41. Nous avons repéré 18 titres de publications relevant du Service de sa création à 1961 .

42. (Québec, province) ministère de la Santé, Division de la nutrition, hygiène maternelle et infantile, Considérations sur les besoins du corps humain et les règles alimentaires (Québec, ministère de la Santé, 1954), $93 \mathrm{p}$. 


\section{FIGURE 2 \\ Manuel de cuisine préparé par la Division de la nutrition pour les camps, 1949}

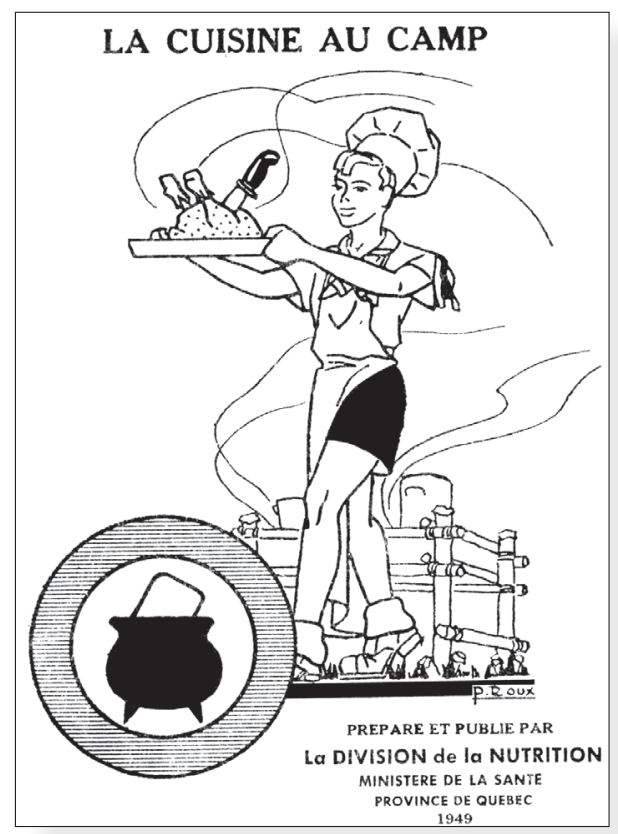

Source: (Québec, province) ministère de la Santé, Division de la nutrition, La cuisine au camp (1949).

aux étudiants en médecine et aux professeurs et élèves d'écoles normales, d'écoles d'infirmières ou d'instituts familiaux. D'autres publications sont conçues pour des organisations qui servent des repas à des groupes de différentes tailles, ce qui renvoie encore à un effort de bonification de l'environnement de l'individu. Un manuel de recettes par exemple vise les camps de vacances (voir figure 2 ) $^{43}$. Une troisième catégorie de publications s'adresse au grand public ${ }^{44}$. Ces documents sont distribués lors de

43. (Québec, province) ministère de la Santé, Division de la nutrition, La cuisine au camp (1949), 81 p. Révisé et réédité en 1961 sous le titre Hygiène et cuisine au Camp. Manuel à l'usage des scouts et des guides, $130 \mathrm{p}$.

44. C'est le cas par exemple d'une série de trois feuillets publiés en 1957 et 1958: (Québec, province) ministère de la Santé, Division de la nutrition, hygiène maternelle et infantile, Conseils aux parents sur la nutrition des enfants (Québec, ministère de la Santé, 1957), 8 p.; Alimentation durant la grossesse (Québec, ministère de la Santé, 1958), 8 p. ; Les légumes, aliments protecteurs de la santé (Québec, ministère de la Santé, 1958), 7 p. Parmi d'autres titres, également: (Québec, province) ministère de la Santé, Division de la nutrition, Les repas au travail (Québec, ministère de la Santé, 1954), 16 p. ; la réédition d'une brochure 
différents événements. Pour 1949 par exemple, il est question de 32253 documents distribués lors de causeries ${ }^{45}$. À cela s'ajoutent des articles soumis à la presse et à certaines revues. Ainsi, une série de textes paraît en 1943 dans la Revue des fermières, organe des Cercles de fermières, sous la thématique "Qui mange bien se porte bien ${ }^{46}$ ».

Le message nutritionnel est véhiculé également à l'aide de conférences devant des auditoires variés comme les tuberculeux d'un établissement, des jeunes suivant un cours de préparation au mariage, des Cercles de Fermières, des Filles d'Isabelle ou les membres d'autres associations, ou encore les mères fréquentant les cliniques de puériculture dans les années $1960^{47}$. Il arrive aussi que des séances soient offertes aux élèves d'écoles ménagères comme en 1944, à Saint-Pascal de Kamouraska ${ }^{48}$. Des "causeries» ou «leçons» sont tenues, des films projetés. Le rapport annuel du ministère de la Santé pour 1951 livre ainsi une liste de quatre «films sonores» et de 15 "films de vues fixes» portant des titres tels "Pourquoi nous mangeons des fruits» ou «Se bien nourrir pour mieux servir ${ }^{49}$.

Des conférences sont livrées dans des couvents, écoles, hôpitaux, instituts familiaux... En 1955 par exemple, des assistances comptant plus de 10100 enfants et 658 enseignants ont été rejointes dans les maisons d'éducation $^{50}$. La radio, et plus tard la télévision, sont utilisées aussi, par exemple en 1943 lorsque cinq causeries sont diffusées sur les ondes, permettant de toucher une plus grande population ${ }^{51}$. Des kiosques sont également préparés pour l'Exposition provinciale de Québec à partir de 1942 (voir figure 3, p. 180). Dans les années 1950, il en va de même pour des expositions liées aux congrès de l'Association des Hôpitaux Catholiques de la Province de Québec, de l'Association Canadienne des Infirmières et des Cercles des Fermières.

Un groupe préoccupe particulièrement les autorités sanitaires, celui des écoliers. Outre l'examen médical, pour lequel Sylvestre rédige un guide

datant des années 1920, avec ajout de considérations nutritionnelles, (Québec, province) ministère de la Santé, Division de la nutrition, Principes d’hygiène (Québec, ministère de la Santé, 1948), 23 p.

45. (Québec, province), ministère de la Santé, Sixième rapport du Ministère de la Santé pour l'année 1949 (Québec, ministère de la Santé, 1950), 65.

46. Revue des Fermières, série d'articles parus dans les numéros de février 1943 à janvier 1944.

47. Consulter à ce propos les rapports annuels de la Division.

48. Ibid., p. 285.

49. (Québec, province) ministère de la Santé, Huitième Rapport..., op. cit., 75.

50. (Québec, province) ministère de la Santé, Douzième Rapport..., op. cit., 83.

51. (Québec, province) ministère de la Santé et du Bien-être Social, Deuxième rapport..., op. cit., 278. 


\section{FIGURE 3}

\section{Kiosque de la Division de la nutrition à l'exposition provinciale, I 942}

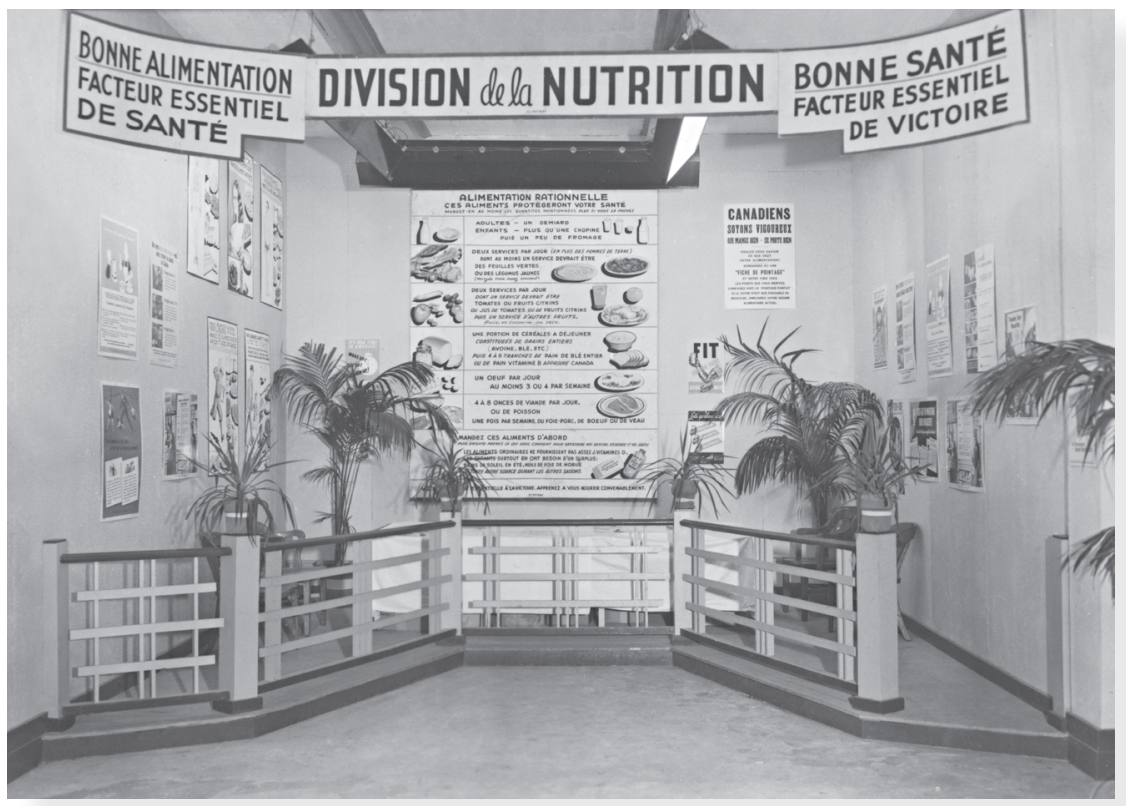

Source: BAnQ, Fonds ministère de la Culture et des Communications, Office du film du Québec, E6, S7, SSI, PI5020. Photographe Raymond Audet.

destiné aux médecins ${ }^{52}$, toute une panoplie de moyens d'intervention vise ce segment de la population. C'est le cas par exemple de concours visant à susciter l'intérêt des écoliers. En 1945, la Division de l'hygiène alimentaire du gouvernement fédéral organise un "concours de pancartes» national récompensé par des certificats d'épargne de guerre à raison de trois gagnants par province ${ }^{53}$. Les élèves sont alors invités à illustrer les cinq groupes des règles alimentaires canadiennes. Un autre concours d'affiches est tenu en 1951 par le Service de santé de la ville de Montréal lors d'une semaine de santé organisée sur les terrains de jeux ${ }^{54}$. La Division

52. J. Ernest Sylvestre, L'examen médical à l'école. Manuel à l'usage du personnel des Unités Sanitaires (Québec, ministère de la Santé, 1960), 31 p.

53. (Canada) ministère de la Santé nationale et du Bien-être social, Rapport annuel du Ministère de la Santé nationale et du Bien-être social pour l'année financière terminée le 31 mars 1946 (Ottawa, Imprimeur du roi et contrôleur de la papeterie, 1947), 63 ; BAnQ, Ministère de l'Éducation, du Loisir et du Sport, Département de l'Instruction publique, Comité catholique du Conseil de l'Instruction publique, correspondance relative au Concours de pancartes dans les écoles, 3 B 019 01-02-003A-01 / 1945 -

54. Adélard Groulx, Rapport du Service de santé (Montréal, Ville de Montréal, 1951), 133. 
utilise aussi les enquêtes alimentaires menées dans les écoles pour sensibiliser les enfants, les parents et les enseignants aux principes d'une saine alimentation $^{55}$. Le contenu de la boîte à lunch des enfants est d'ailleurs l'objet de bien des attentions. Ainsi, en 1946, dans le cadre d'un concours, les institutrices accorderont des points selon la valeur nutritive des aliments apportés à l'école ${ }^{56}$. Au début des années 1960, une enquête annuelle est lancée concernant le contenu des boîtes à lunch, à l'occasion de laquelle les écoliers reçoivent de l'information, répondent à des questionnaires et sont conseillés sur la façon d'améliorer leur alimentation ${ }^{57}$. À travers les enfants, on espère aussi rejoindre les parents.

\section{LES CONSIDÉRATIONS LIÉES AUX ALIMENTS}

Cette information nutritionnelle s'accompagne souvent de messages d'autre nature. Il a été montré que dans les manuels scolaires préparés sous l'autorité du clergé catholique jusqu'en 1960, les avis donnés sur l'alimentation appuient des vues conservatrices, en célébrant le rôle de la femme au foyer et en promouvant le nationalisme traditionaliste avec ses valeurs religieuses et ruralistes ${ }^{58}$. Il a été aussi montré que les concepteurs des règles alimentaires canadiennes comme ceux des manuels scolaires font valoir les gains en productivité d'une population mieux nourrie, en mettant toutefois l'accent sur les responsabilités individuelles plutôt que collectives, ce qui justifie les projets éducatifs au détriment de réformes sociales assurant un minimum de revenus aux familles ${ }^{59}$.

Pourtant, les interventions publiques de la Division de la nutrition ne suivent qu'en partie ces modèles, comme le montre l'analyse du contenu de 37 documents: onze brochures ou livrets publiés par la Division ${ }^{60}$, onze

55. Dans un texte expliquant la méthodologie à suivre pour une enquête, il est indiqué : «Une enquête sur les habitudes alimentaires est un moyen pratique d'intéresser les écoliers à l'hygiène alimentaire et permet de découvrir quelles améliorations il y aurait lieu d'apporter au régime alimentaire des enfants ". Dans BAnQ, MSSS, Enquêtes sur la nutrition, 7 B 012 06-05-002A-01 / 1959, Méthode d'enquête sur les habitudes alimentaires, 1959, 1.

56. (Québec, province) ministère de la Santé et du Bien-être Social, Troisième rapport..., op. cit., 308.

57. (Québec, province) ministère de la Santé, Dix-septième Rapport du Ministère de la Santé pour l'année 1960 (Québec, ministère de la Santé 1961), 103. Voir aussi les rapports des années suivantes.

58. Caroline Durand, "Rational Meals for the Traditional Family: Nutrition in Quebec School Manuals, 1900-1960", dans Franca Iacovetta, Valerie J. Korinek et Marlene Epp, dir., Edible Histories, Cultural Politics, op. cit., 109-127. Voir aussi Caroline Durand, Le laboratoire domestique de la machine humaine: la nutrition, la modernité et l'État québécois, 1860-1945, thèse de doctorat (histoire), Université McGill, 2011.

59. I. Mosby, op. cit.

60. (Québec, province), ministère de la Santé et du Bien-être social, Division de la nutrition, hygiène maternelle et infantile, L'alimentation des enfants. Un moyen de défense contre la tuberculose (Québec, ministère de la Santé et du Bien-être social 1941), 15 p.; (Québec, province) ministère de la Santé, Division de la 
articles de revues dont elle est l'auteure ${ }^{61}$, treize articles de journaux rendant compte de son action, un texte de conférence de J.-E. Sylvestre et une lettre donnant des directives ${ }^{62}$. Il est vrai que la situation financière des familles est peu abordée. On préfère proclamer que généralement, les gens mangent à leur faim, mais ne savent pas choisir les aliments. Il est vrai aussi que la responsabilité de diffuser et d'utiliser les conseils échoit souvent aux mères. Mais cette responsabilité ne leur est pas exclusive, loin de là. Elle relève aussi d’autres acteurs. Généralement, c’est la personne à qui s'adresse l’imprimé ou la conférence qui est désignée responsable. Ainsi les enseignants, masculins et féminins, se doivent d'éveiller leurs élèves aux principes d’une saine nutrition. Les infirmières et médecins, spécialement des unités sanitaires, devraient saisir toutes les occasions d'en parler. Les scouts et les guides devraient ramener à la maison les connaissances acquises au camp d'été. Devant un large public, on s'adresse tout aussi souvent aux parents ou aux pères et mères qu'aux femmes spécifiquement. Les pères peuvent être interpellés, comme dans une brochure de la Division en 1948 où on leur dit: «la santé et le bonheur de votre femme et de vos enfants dépendent de vous. SACHEZ LES LEUR DONNER ${ }^{63}$ !».

Les considérations religieuses sont quasiment absentes. Sauf exception, elles surgissent dans les années précédant de peu la Révolution tranquille,

nutrition, Principes d'hygiène, op. cit.; (Québec, province) ministère de la Santé, Division de la nutrition, $L a$ cuisine au camp, op. cit.; (Québec, province) ministère de la Santé, Division de la Nutrition, Les repas au travail (1954), 16 p.; (Québec, province) ministère de la Santé, Division de la nutrition, hygiène maternelle et infantile, Considérations sur les besoins du corps humain et les règles alimentaires (Québec, ministère de la Santé, 1954, autres éditions aussi), 93 p.; (Québec, province) ministère de la Santé, Division de la nutrition hygiène maternelle et infantile, Manuel de diétothérapie (Québec, ministère de la Santé, 1957), 54 p. ; Québec, province), ministère de la Santé, Division de la nutrition, hygiène maternelle et infantile, Conseils aux parents sur la nutrition des enfants, op. cit.; (Québec, province), ministère de la Santé, Division de la nutrition, hygiène maternelle et infantile, Alimentation durant la grossesse, op. cit.; (Québec, province), ministère de la Santé, Division de la nutrition, hygiène maternelle et infantile, Les légumes, aliments protecteurs de la santé, op. cit.; (Québec, province) ministère de la Santé, Division de la nutrition, hygiène maternelle et infantile, Hygiène et cuisine au Camp..., op. cit.; (Québec, province) ministère de la Santé, Service de la nutrition, Manuel de Diétothérapie (Québec, ministère de la Santé, 1965, 4e édition, révisée), 104 p.

61. Neuf de ces articles ont été publiés dans la Revue des Fermières dans les éditions suivantes: mai-juin 1942, avril 1943, mai-juin 1943, juillet-août 1943, septembre 1943, octobre 1943, novembre 1943, décembre 1943 et janvier 1944. Deux proviennent de la revue L'enseignement primaire, dans les numéros d'octobre et de décembre 1947.

62. BAnQ, ministère de la Santé et des Services sociaux, Enquêtes sur la nutrition, 7B012 06-05-002A01/1936-57, J. Ernest Sylvestre, «La prévention des accidents et des maladies» [texte d'une communication au congrès des infirmières d'industrie tenu à Québec en 1955], 25-26 février 1955; BAnQ, Fonds ministère de l'Education, du Loisir et du Sport, Département de l'Instruction publique, Comité catholique du Conseil de l'Instruction publique, 3B019 01-02-003A-01/1945, lettre de Victor Doré, surintendant de l’Instruction publique aux Titulaires des écoles primaires élémentaires, sur demande de la Division de la nutrition, 1945.

63. (Québec) ministère de la Santé, Division de la nutrition, Principes d’hygiène, op. cit., 3. 
alors que l'action du clergé dans la société est remise en question. On trouve ainsi dans la deuxième édition d'une publication pour les scouts et les guides en 1961, une référence à Dieu inexistante dans l'édition originale de $1949^{64}$. L'ajout est lié à la collaboration d'une missionnaire laïque, ancienne guide et cheftaine. Par ailleurs, lorsqu'il y a considérations religieuses, elles ne dépassent pas la partie introductive.

Les appels au nationalisme canadien-français sont encore plus rares. Un seul exemple a été repéré où, lors d'une conférence devant la société Saint-Jean-Baptiste, il a été question, d'après le journaliste relatant l'événement, de restaurer les vertus anciennes d'une population frappée de déchéance physique ${ }^{65}$. Certes, en temps de guerre, de saines habitudes alimentaires sont présentées comme un préalable à la victoire, mais on parle alors de la nation canadienne.

Après la guerre, on tend à abandonner l'évocation des gains collectifs que procure une saine alimentation, pour faire plutôt valoir des avantages individuels. Les références à la prospérité et à la force de la nation, très présentes dans le discours d'hygiène publique de la première moitié du siècle, s'effacent. Le rapport à la nation demeure tout de même parfois présent, mais sous une autre forme, par l'exhortation à la formation de bons citoyens, solides physiquement et mentalement. S'adressant aux parents en 1957, on écrit : "vous ne ferez pas qu'améliorer leur état de nutrition, vous en ferez des citoyens en état de rendre le maximum des services que vous et la société êtes en droit d'attendre d'eux ${ }^{66}$ ». Les références au bonheur et au succès personnel apparaissent régulièrement, de même que les préoccupations pour le développement de l'intelligence, pour la vivacité intellectuelle opposée aux difficultés d'attention à l'école. Dans un article destiné aux enseignantes du primaire, on affirme par exemple: «un enfant bien alimenté est facilement heureux, il apprend vite de nouvelles choses et remporte des succès ${ }^{67} »$. Et l'alimentation est de plus en plus présentée aux côtés d'autres habitudes de vie qui, ensemble,

64. (Québec) ministère de la Santé, La division de la nutrition, La cuisine au camp, op. cit., 81 p. ; (Québec) ministère de la Santé, Division de la nutrition, hygiène maternelle et infantile, Hygiène et cuisine au camp, op. cit., 8-9.

65. [s.a.], «Nécessité d'une saine nutrition», Journal non identifié, probablement 1942 ou 1943, tiré d'un recueil d'articles, dans BAnQ, ministère de la Santé et des Services sociaux, Nutrition, Coupures de journaux, 7 B 012 01-02-005A-01/1941-65.

66. (Québec), Ministère de la Santé, Division de la Nutrition, Hygiène maternelle et infantile, Conseils aux parents sur la nutrition des enfants, op. cit., 8.

67. Division de la Nutrition au ministère de la Santé, "Le "LUNCH" à l'école. Deux cents chances de rendre service!», L'enseignement primaire, 7, 2, octobre 1947, endos. 
contribuent à dessiner tout un mode de vie idéal: activité physique et repos, propreté et maintien, ambiance gaie et agréable...

D'autres thématiques sont stables et traversent toutes les périodes: vigueur, énergie et force ainsi que leurs contraires, fatigue et faiblesse, sont des constantes, comme la croissance et la réparation de l'organisme de même que sa protection devant les maladies. D'autres thèmes enfin, communément mentionnés dans les décennies ultérieures, ne se profilent encore qu'exceptionnellement, tels l'allongement de la vie et l'amélioration de l'apparence physique, bien que cette dernière ressorte parfois.

\section{LA POLITIQUE QUÉBÉCOISE DE NUTRITION ET LES GRANDS PROGRAMMES}

Les actions évoquées se développent à partir de la fin des années 1930 et se prolongent dans les années 1960. Mais des remises en question surgissent alors et la décennie 1970-1980 marque un tournant majeur en matière de politiques nutritionnelles au Québec.

Le service de la nutrition est éliminé en 1970 et ses responsabilités partagées entre d'autres services. À la suite d'une vaste enquête sur l'alimentation des Canadiens effectuée par le gouvernement fédéral au début de la décennie, un rapport est publié en 1975 pour le Québec, attirant l'attention sur divers problèmes dont l'embonpoint et la consommation exagérée de matières grasses et de sucre ${ }^{68}$. Dans la foulée de cette enquête, le ministère des Affaires sociales forme dès avril 1973 un comité chargé de définir une politique québécoise de nutrition ${ }^{69}$. Le comité est composé de quatre représentants du ministère, à savoir deux diététistes-nutritionnistes et deux membres du service des études épidémiologiques, ainsi que de plusieurs universitaires reconnus pour leur expertise en nutrition. Il produit le rapport Une politique québécoise en matière de nutrition ${ }^{70}$, rendu public en septembre $1977^{71}$. Basé sur l'enquête canadienne en ce qui a trait à l'évaluation de la situation, il dénonce les coûts associés au traitement des maladies liées à une mauvaise alimentation, ce qui réintroduit les préoccupations d'ordre collectif dans un contexte où vient de se mettre

68. (Canada) Bureau des sciences de la nutrition, Nutrition Canada: compte-rendu de l'étude menée au Québec (Ottawa, MSNBES, 1975), 172 p.

69. BAnQ, Ministère de la Santé et des Services sociaux, Santé au travail et environnementale, 2 B 012 03-01-001B-01 / 1978, Comité spécial du CASF sur la nutrition, Avis et recommandations concernant les objectifs et les moyens d'action de la politique québécoise en matière de nutrition, décembre 1978, p. 4.

70. (Québec, province) Service des études épidémiologiques, Une politique québécoise en matière de nutrition, op. cit.

71. Comité spécial du CASF sur la nutrition, Avis et recommandations..., op. cit., 3. 
en place l'assurance maladie d'État. Lors de la conférence de presse annonçant la nouvelle politique, le ministre Denis Lazure rappelle d'ailleurs que ce sont "principalement l'évolution inquiétante des habitudes alimentaires de la population et le coût élevé du traitement des maladies associées à ces habitudes préjudiciables à la santé qui [1’ont] incité à élaborer une politique en matière de nutrition ${ }^{72}$ ».

Un comité d'implantation de cette politique, composé de fonctionnaires du ministère des Affaires sociales, est alors chargé de définir une stratégie d'action pour les dix années à venir. Son premier programme d'envergure sera une campagne de "contre-publicité » à la radio et à la télévision, dont l'objectif est, comme sa désignation le suggère, de contrer une publicité existante, celle d'une industrie alimentaire plus soucieuse de profits que de la santé de ses clients. Un autre comité spécial dédié à la nutrition est nommé en 1978, consultatif et relevant du Conseil des Affaires sociales et de la Famille (CASF). Il regroupe plusieurs secteurs d'activité, des professionnels de la santé (médecins, infirmières, diététistes) et des représentants des milieux agricole et scolaire ${ }^{73}$. Comme le ministre Lazure l'explique lors d'une rencontre d'ouverture, il vise à susciter la collaboration des milieux professionnels impliqués le plus directement dans la nutrition des Québécois. Ajoutons à cela un comité interministériel devant régler la nécessaire coordination du travail de plusieurs ministères touchés par la politique de nutrition, à savoir les ministères des Affaires sociales, de l'Éducation, de l'Agriculture, des Finances et de l'Industrie et du Commerce $^{74}$ ainsi que l'Office de la Protection du consommateur et le Haut Commissariat à la jeunesse, aux loisirs et aux sports.

Il ressort clairement de cet enchaînement de rapports et de comités que la nutrition est devenue une question d'importance pour plusieurs et qu'elle sera chaudement débattue. La multiplication des comités s'accompagnera d'ailleurs de tensions quant aux rôles et aux responsabilités de chacun, comme l'illustre une lettre de la secrétaire du Comité d'implantation de la

72. Id.

73. BAnQ, Ministère de la Santé et des Services sociaux. Conseil des Affaires sociales et de la Famille, 7 D 025 03-01-001A, notes biographiques concernant les membres du comité spécial du CASF sur la nutrition, c1978; BAnQ, Conseil de la santé et du bien-être, 7 D 022-02-05-001B-01, Comité spécial du CASF sur la nutrition, Compte rendu de réunion, 15 septembre 1978, 8 p.

74. BAnQ, Fonds Jean Garon, série Ministère de l'Agriculture, des Pêcheries et de l'Alimentation, 1999-04-013/397, lettre de Denis Lazure, ministre des Affaires sociales à Jean Garon, ministre de l'Agriculture, Sujet: Implantation de la politique québécoise en matière de nutrition, 31 mai 1978; BAnQ, Conseil de la santé et du bien-être, 7 D 022-02-05-001B-01, Comité spécial du CASF sur la question de la nutrition, Compte rendu, Annexe 1: Rencontre de M. Aubert Ouellet, sous-ministre adjoint, avec le comité spécial du CASF, 24 mai 1978, 6 p. 
politique québécoise en matière de nutrition au secrétaire du CASF ${ }^{75}$. La formation même d'un comité interministériel vise sans doute en partie à résoudre les questions de territoire qui se posent lors de la mise en œuvre de politiques affectant les champs d'action de plusieurs ministères.

Ce type de situation où divers ministères, chacun poursuivant ses objectifs propres, ont à collaborer tout en se disputant parfois le champ d'intervention de la nutrition, peut être illustré à l'aide du programme de distribution de lait dans les écoles. L'initiative de ce programme ne provient pas du ministère responsable de la santé qui met plutôt l'accent sur la promotion d'une consommation équilibrée et dite rationnelle d'aliments représentatifs de chacun des grands groupes alimentaires recommandés dans le guide canadien. Il ne fait pas passer un aliment devant les autres. Le ministère qui a charge de l'agriculture procèdera autrement en 1977.

Jusqu'alors, la distribution de lait dans certaines écoles s'était faite sous l'égide d'autorités scolaires locales, par exemple à Montréal depuis 1934 par la Commission des écoles catholiques de Montréal, ou en Mauricie et dans le Bas-Saint-Laurent, souvent avec le soutien de fondations et de clubs sociaux (Progress Club dans les milieux anglophones, Richelieu, Lions, Kiwanis, Chevaliers de Colomb, Filles d'Isabelle, etc., chez les francophones), de même que la participation sous diverses formes de producteurs laitiers et de laiteries ${ }^{76}$. Mais les milieux agricoles et l'industrie laitière en viennent dans les années 1950 à militer pour un programme à l'échelle provinciale. Au congrès de l'Association des industriels laitiers en 1954, la distribution de lait dans les écoles et les propos d'une nutritionniste à cet égard suscitent beaucoup d'intérêt. Au congrès de l'année suivante, l'association se prononce en faveur d'un programme alliant producteurs, industriels, commissions scolaires et fondations privées.

$\mathrm{Au}$ fil des ans, l'industrie laitière québécoise obtient divers appuis: conférence interprovinciale de l'Agriculture de 1955, Chambre de com-

75. BAnQ, Ministère de la Santé et des Services sociaux. Santé au travail et environnementale, 2 B 012 03-01-001B-01/1978, lettre d'Isabelle Giroux, secrétaire du Comité d'implantation de la politique québécoise en matière de nutrition à André Escojido, secrétaire du Conseil des Affaires sociales et de la Famille, 12 juillet 1978 .

76. Les informations de ce paragraphe proviennent des documents suivants: Alcide Courcy, Résumé du rapport du Comité d'étude de la distribution du lait dans les écoles de la province de Québec (Québec, ministère de l'Agriculture et de la Colonisation, 1962), 18 p.; une version préliminaire annotée du même document, BAnQ, MSSS, Bureau du sous-ministre, Montreal Diet Dispensary; L'Association des Industriels Laitiers de la province de Québec Inc., Plan pratique de distribution généralisée du lait dans les écoles de la province de Québec (Montréal, L’Association des Industriels Laitiers de la province de Québec Inc., 4 avril 1962), 37 p. ; «Le Lait-Écolier dans nos écoles de Trois-Rivières et du Cap-de-la-Madeleine », Le Nouvelliste, vendredi 20 septembre 1963 , p. 10. 
merce de la Province de Québec, Fédération canadienne de l’agriculture, Conseil national de l'industrie laitière. En 1960, l'Union catholique des cultivateurs et l'Association des industriels laitiers déposent une requête conjointe au ministère de l'Agriculture et de la Colonisation afin que soit mise sur pied une distribution à moitié prix avec l'aide financière du gouvernement. Un comité d'étude de la distribution de lait est ensuite formé sous l'autorité du ministre. Il sera présidé par J.-E. Sylvestre accompagné de quatre agronomes et d'un inspecteur rattaché au Conseil de l'instruction publique. À la requête du comité, divers mémoires sont soumis, notamment par l'Union catholique des cultivateurs et la Fédération des syndicats de producteurs de lait.

Un autre mémoire, celui de l'Association des industriels laitiers, présenté en 1962, propose un plan de distribution généralisée dans les écoles, recourant au support financier de l'État, à titre d'action supplétive à celle des acteurs privés. Pour légitimer son projet, l'association fait valoir qu'il s'agit "d'une entreprise à portée sociale, de promotion de la santé publique beaucoup plus [que d'un] plan de restauration ou de consolidation économique de l'industrie laitière ${ }^{77}$ ». Le rapport du comité ministériel constate une surproduction de l'industrie laitière, signale qu' "On considère le marché qu'offrent les écoles comme le principal débouché pour les surplus agricoles", et calcule les retombées pour le marché laitier d'une distribution généralisée en se basant sur l'exemple des États-Unis. Au nom à la fois de la santé des enfants et du développement de l'industrie, il propose une distribution généralisée de lait à bas coût, supervisée par un administrateur et un comité consultatif où siégeraient des représentants des ministères du Bien-être social et de la Famille, de la Santé et de l'Agriculture ainsi que de l'Instruction publique. Le projet ne sera toutefois repris que quinze ans plus tard.

Entre-temps, la question de l'alimentation dans les écoles donne lieu à des enquêtes et est débattue ${ }^{78}$. Les organisations de producteurs laitiers maintiennent leurs pressions sur le gouvernement provincial ${ }^{79}$. Une organisation de consommateurs, l'Association coopérative d'économie familiale de Québec, réclame également un programme de distribution de lait

77. Ibid., 1.

78. Voir par exemple «Les diététistes réclament la responsabilité des services alimentaires dans les écoles ", Le Devoir (20 février 1968): 11; (Québec) Ministère des Affaires sociales, Enquête alimentaire en milieu scolaire au Québec (niveau secondaire) (Québec, Ministère des Affaires sociales, 1972), 67 p.

79. Michel Morin, «Une pinte de lait coûtera 49 cents, le $1^{\text {er }}$ janvier», Le Soleil (2 octobre 1974): A-3. 
dans les écoles ${ }^{80}$. Surtout, le monde agricole est en émoi en raison de changements des politiques fédérales affectant la production laitière et les prix du lait ${ }^{81}$. En 1975, le "marché est inondé d'une surabondance de produits $^{82}$ », ce qui mène l'année suivante à des réductions majeures de quotas qui affectent particulièrement le Québec. Les producteurs laitiers sont furieux et ils organisent une manifestation mémorable à Ottawa.

Lorsque le Parti québécois prend le pouvoir en 1976, les agriculteurs québécois sont donc nettement insatisfaits des politiques canadiennes, ce qui constitue une situation propice à une démonstration de ce que pourrait faire un gouvernement du Québec si ce dernier devenait souverain. Jean Garon, ministre de l'Agriculture, met en place dès l'année suivante un programme de distribution de lait dans une partie des écoles de niveau primaire, lequel programme sera étendu par la suite à toutes les écoles ${ }^{83}$. Le financement gouvernemental favorise ainsi l'industrie laitière et l'écoulement des surplus de la production. Le ministre Garon fonde tout de même son action en bonne partie sur une argumentation d'ordre sanitaire et son ministère produit sa propre documentation éducative distribuée aux enseignants et aux écoliers. La revue Gobe-Lait créée pour l'occasion adopte une thématique à saveur de science-fiction jugée accrocheuse pour les élèves. Elle promeut, aux côtés du lait qui domine nettement ses pages, l'usage d'autres aliments (voir figure $4^{84}$ ).

Le ministère de l'Éducation collabore notamment en fournissant des équipements de refroidissement. Le ministère responsable de la santé, toutefois, ne semble avoir été que marginalement consulté ${ }^{85}$. Des montants dont n'aurait jamais pu rêver la Division de la nutrition, à savoir environ deux millions de dollars pour la première année d'implantation et plus les années suivantes ${ }^{86}$, sont ainsi consacrés par le gouvernement à l'achat et à la promotion du lait, au nom de la santé des écoliers et de

80. Denis Fortin et Marc Roland, La gratuité du lait dans les écoles : une lutte de consommateurs? (Québec, Groupe de Recherche en Action Populaire, Cahier 6, 1981), 22 p.

81. Erin Scullion, La Commission canadienne du lait. 40 ans d'histoire (Ottawa, La Commission canadienne du lait, 2006), p. 58-63.

82. Ibid., p. 58.

83. La mise en place du programme est bien documentée à BAnQ-Québec, dans le fonds Jean Garon, Ministère de l'Agriculture, des Pêcheries et de l'Alimentation (FJG).

84. (Québec, province) ministère de l'Agriculture et de l'Alimentation, Le Gobe-lait, c1978, 1.

85. Au moment de l'annonce du programme lait-école, Jean Garon forme un comité où siègent notamment des représentants du ministère des Affaires sociales et de l’Éducation. Voir FJG, 1999-04013/358/1979, "Comité consultatif pour le programme lait-école», 1977.

86. FJG 1999-04-013/358/ 1979, (Québec, province) ministère de l’Agriculture, Cabinet du ministre, Déclaration du ministre pour le lancement du lait-école. Créer de meilleures habitudes alimentaires chez les enfants du Québec, 19 septembre 1977, 2 p. 
FIGURE 4

Page titre d'un numéro de la revue Gobe-Lait

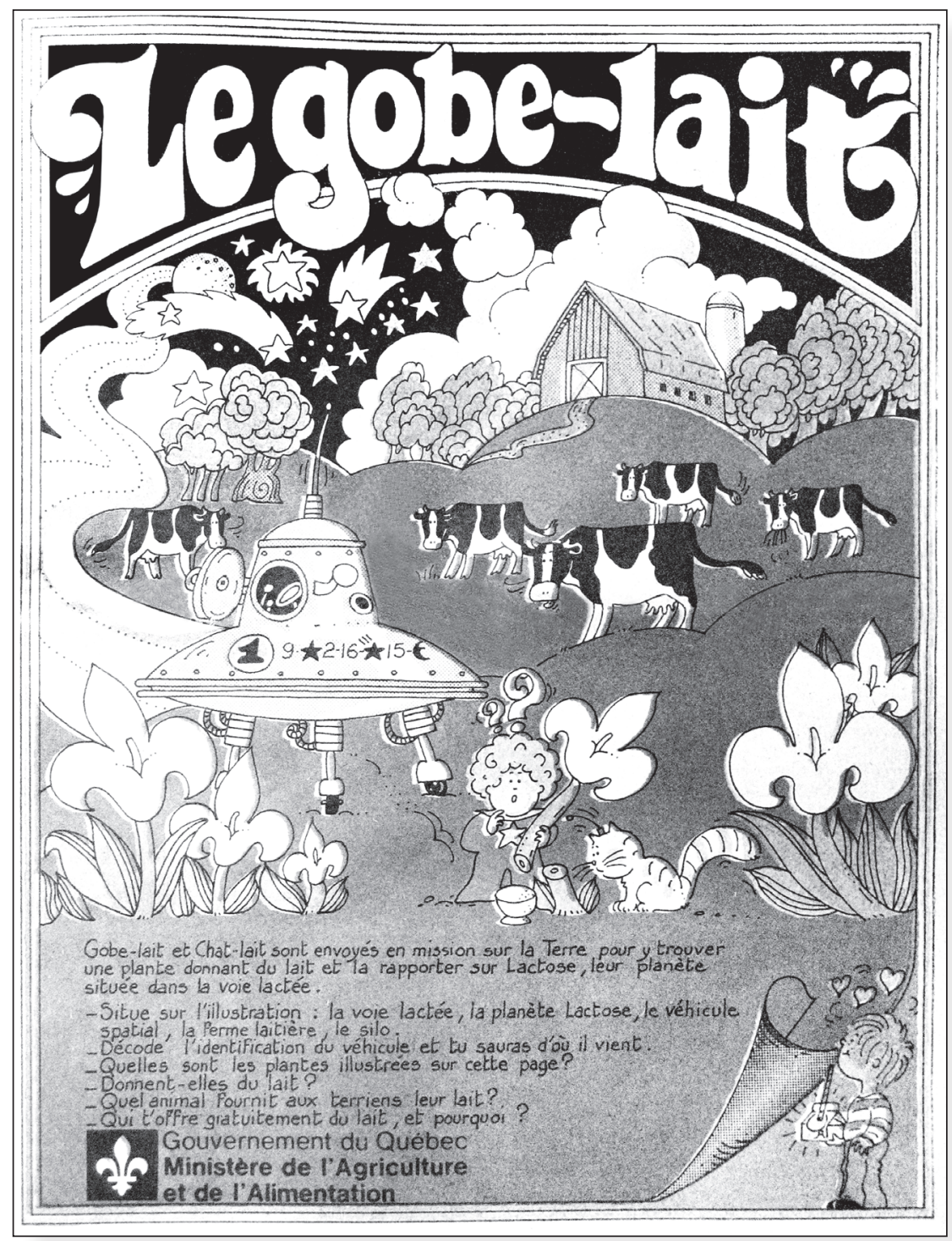

Source: (Québec, province) ministère de l'Agriculture et de l'Alimentation, Le Gobe-lait, cl978. 
l'acquisition de saines habitudes, en même temps que de la correction de problèmes sociaux laissant certains enfants sans alimentation appropriée. Ce programme, contesté quant à ses réels apports à la santé de la population $^{87}$, constitue bel et bien une mesure sociale élargissant les possibilités de choix alimentaire pour l'enfant d'école.

Comme l'illustre la distribution de lait en 1977, les chevauchements entre ministères se multiplient. Cela se produit de surcroît alors que d'autres acteurs sociaux interviennent dans le champ de l'alimentation. Par exemple, les organismes de protection du consommateur récemment créés s'intéressent aux paniers de provisions afin d’indiquer aux Québécois la meilleure façon de se nourrir au prix le plus avantageux ${ }^{88}$. La multiplication des discours nutritionnels vient également complexifier la situation, par exemple avec la large diffusion médiatique des idées des naturistes et naturopathes à partir des années $1960^{89}$. Au moment où la nutrition s'immisce ainsi dans les grands débats publics, il se trouve qu'au Québec, les structures d'intervention antérieures ne tiennent plus. En effet, le chambardement des systèmes d'éducation, de la santé et des services sociaux, avec le transfert de responsabilités de l'Église à l'État, court-circuite les réseaux traditionnels jusqu'alors utilisés pour la transmission de l'information nutritionnelle.

Bref, la nutrition est devenue un sujet chaudement débattu, alors que divers ministères doivent coordonner leur action, et qu'interviennent d'autres acteurs, incluant des groupes de pression de producteurs et de consommateurs.

\section{CONCLUSION}

Les dernières années de la décennie 1970 marquent un nouveau départ pour la nutrition au Québec, alors que le gouvernement se dote d'une politique globale et entreprend une action plus énergique qu'auparavant, sollicitant la collaboration de multiples acteurs. Les efforts antérieurs, en bonne partie dénigrés ou oubliés, méritent pourtant que l'on s'y arrête.

87. Des doutes sur son utilité sont émis dès 1977 par le docteur Claude C. Roy qui prend à témoin des études faites en Angleterre et aux États-Unis. FJG, 1999-04-013/307/ 1977-78, lettre de Claude C. Roy, professeur de pédiatrie, chef du Service de Pédiatrie de l'hôpital Sainte-Justine et président du Comité de Nutrition de la Société Canadienne de Pédiatrie à Maurice Bouchard, président de l'Association des Pédiatres de la Province de Québec, 15 septembre 1977.

88. BAnQ, Conseil de la santé et du bien-être, 7 D 022-02-05-001B-01, Comité spécial du CASF sur la nutrition, Compte rendu, 30 mars 1979, 8 p.

89. Catherine Carstairs, "The Granloa High: Eating Differently in the Late 1960s and 1970s », dans F. Iacovetta, V. J. Korinek et M. Epp, dir., Edible Histories Cultural Politics..., op. cit., 305-325. 
L'examen du travail de la Division de la nutrition montre que dès la fin des années 1930, on tente d'amener la population à améliorer ses habitudes alimentaires: des enquêtes sont menées; une formation est donnée à des éducateurs, à des médecins, à des infirmières et à d'autres personnes actives en hygiène publique; des outils de promotion sont conçus et des brochures distribuées en grand nombre; la presse écrite, la radio et la télévision sont mises à contribution; des conférences sont données, des films projetés... Si la vaste majorité des démarches ainsi entreprises sont à caractère éducatif, certaines visent déjà à améliorer l'environnement de l'individu: des conseils sont donnés aux établissements scolaires et hospitaliers ainsi qu'à des entreprises pour qu'ils rehaussent la qualité sanitaire de leurs services alimentaires, des publications visent les organisations qui servent des repas à de grands groupes et un programme provincial de distribution de lait prendra en 1977 le relais de programmes locaux dispersés. Il s'agit là d'actions spécifiques à l'alimentation, auxquelles il faut ajouter celles à plus large spectre adoptées durant la Crise, la Guerre puis l'après-guerre, garantissant un revenu minimum et donc une meilleure capacité d'achat et de choix des aliments pour plusieurs.

Jusqu'au milieu de la décennie 1960, l'effort éducatif gouvernemental met l'accent sur le rôle des mères et des ménagères, mais en interpellant tout aussi bien d'autres acteurs. Contrairement à ce qui a été observé dans les manuels scolaires, la religion et le nationalisme traditionaliste québécois imprègnent peu cette information. Toutefois, comme la Division de la nutrition fait transiter cette information par d'autres organisations, ces dernières peuvent lier aux avis nutritionnels la promotion de leurs propres vues et valeurs. Il est à noter également, dans l'après- guerre, la montée des considérations individualistes dans l'argumentation. Dans les décennies antérieures, depuis le $\mathrm{XIX}^{\mathrm{e}}$ siècle, les promoteurs de l'hygiène publique avaient souvent mis de l'avant des avantages collectifs, ce qui permettait de légitimer l'action de l'État dans la pensée libérale de l'époque. Ce type d'arguments, éclipsés durant les décennies 1950 et 1960, ressurgira par la suite, quoique sous une forme nouvelle, au moment où l'État québécois aura parachevé sa réforme du système de santé et des services sociaux dans une perspective d'État-providence.

Il demeure difficile d'évaluer l'envergure et surtout l'efficacité des moyens consacrés par le gouvernement à l'éducation nutritionnelle de la population. Certes, la Division de la nutrition n'a que bien peu de ressources. Mais d'autres acteurs sont actifs, comme les unités sanitaires ou d'autres ministères, ou encore des organismes caritatifs. Les établisse- 
ments d'enseignement, les commissions scolaires entrent en jeu. Il demeure en fait difficile d'évaluer dans quelle mesure l'éducation à l'hygiène alimentaire rejoint la population. Il faut ajouter à cela que déjà dans les années 1960, le discours des organismes publics parvient à la population noyé dans un enchevêtrement d'autres discours nutritionnels, aux côtés de la publicité envahissante de l'industrie alimentaire. Un examen comparé de ces discours entrecroisés s'impose, si l'on veut comprendre les lignes de force de l'argumentaire nutritionnel auquel la population est effectivement exposée. 